\title{
BMJ Open Knowledge, attitude and current practices of pregnant women towards group B streptococcus screening: cross- sectional study, Al-Madinah, Saudi Arabia
}

\author{
Amer Alshengeti (D) , ${ }^{1}$ Amjad Alharbi, ${ }^{2}$ Shahad Alraddadi, ${ }^{3}$ Abdulsalam Alawfi (D) , ${ }^{1}$ \\ Bushra Aljohani ${ }^{4}$
}

To cite: Alshengeti $A$, Alharbi A, Alraddadi S, et al. Knowledge, attitude and current practices of pregnant women towards group $B$ streptococcus screening: crosssectional study, Al-Madinah, Saudi Arabia. BMJ Open 2020;10:e032487. doi:10.1136/ bmjopen-2019-032487

- Prepublication history and additional material for this paper are available online. To view these files, please visit the journal online (http://dx.doi. org/10.1136/bmjopen-2019032487).

Received 29 June 2019 Revised 13 January 2020 Accepted 14 January 2020

Check for updates

(C) Author(s) (or their employer(s)) 2020. Re-use permitted under CC BY-NC. No commercial re-use. See rights and permissions. Published by BMJ.

${ }^{1}$ Pediatric Department, College of Medicine, Taibah University, Madinah, Saudi Arabia

${ }^{2}$ Pediatric Department, Madinah Maternity and Children Hospital, Madinah, Saudi Arabia

${ }^{3}$ Radiology Department, Security Forces Hospital, Riyadh, Saudi Arabia

${ }^{4}$ Obstetrics and Gynecology Department, Madinah Maternity and Children Hospital, Medinah, Saudi Arabia

Correspondence to

Dr Amer Alshengeti; aalshengeti@dal.ca

\section{ABSTRACT}

Aims Group B streptococcus (GBS) is one of the most frequent bacterial pathogens causing invasive infections in neonates. It can be transmitted from colonised mother to neonates around delivery. Screening strategies for GBS during pregnancy include either universal culture-based or risk-based screening. The present study aimed to assess the knowledge, attitude and current practices of pregnant women towards GBS screening in Al-Madinah City, Saudi Arabia.

Methods A hospital-based cross-sectional study was conducted at Madinah Maternity and Children Hospital, AlMadinah, Saudi Arabia, from May to July 2018. Participants were recruited from postnatal wards. Participants were interviewed using a previously published validated survey that was divided into the following domains: demographic data, knowledge, experience and attitude towards different GBS screening strategies.

Results A total of 377 out of 450 women (response rate $83.7 \%$ ) were enrolled. The results showed that the overall mean knowledge score of the pregnant women towards GBS screening was $59.8 \%$. Majority of the women (66.8\%) were not aware of the GBS bacterium, while $86.5 \%$ of them had never been informed of GBS risk assessment during their pregnancies. The mean knowledge score among women who were aware of GBS (62.8\%) was significantly higher than that among women who were not $(58.4 \%)(p=0.015)$. However, majority of the pregnant women $(61.8 \%)$ showed preference for universal culturebased GBS screening strategy over risk-based strategy. Conclusion The study results have concluded that the level of awareness and knowledge about GBS among pregnant women were relatively poor; however, majority of the pregnant women prefer universal culture-based screening.

\section{INTRODUCTION}

Group B streptococcus (GBS) (also known as Streptococcus agalactiae) is a Gram-positive bacterium that colonises the human gastrointestinal and genital tracts. GBS is the most common bacterial pathogen causing invasive
Strengths and limitations of this study

- In Saudi Arabia, there are no national guidelines on GBS screening strategy during pregnancy (ie, universal culture-based vs risk-based).

- To our knowledge, this study is the first study conducted in Saudi Arabia to assess pregnant women's knowledge, attitude and current practices regarding GBS screening in Saudi Arabia.

- The study results are limited because it was conducted in a postnatal department in only one hospital of Al-Madinah, Saudi Arabia.

- A larger sample size and more hospitals are recommended for future studies.

infections in newborn infants during the first week of life. ${ }^{1}$ Maternal colonisation has been found to be a major risk factor for invasive GBS disease in neonates. ${ }^{2}$ Approximately $20 \%-30 \%$ of pregnant women are colonised with GBS in the vagina, rectum or both. ${ }^{3}$ Invasive neonatal GBS infection can be described as either early-onset sepsis (EOGBS) (ie, occurs in the first week of life) or late-onset sepsis (ie, occurs after the first week of life). ${ }^{4}$ The rate of peripartum transmission of GBS to newborns of colonised women is approximately $50 \%$, and about $1 \%-2 \%$ of the neonates born to these mothers develop EOGBS. $^{5}$

The case fatality rate for EOGBS is approximately 22\% among Africans, $11 \%$ among Americans and 7\% among the European population. ${ }^{6}$ EOGBS commonly presents with sepsis without focus in $80 \%-85 \%$ and pneumonia without sepsis in $10 \%$ or both in $35 \%-50 \%$, while late-onset GBS infection is more likely to present with meningitis and septicaemia $^{6}$ 
The risk of EOGBS reduces with the use of intrapartum antibiotic prophylaxis (IAP). ${ }^{7}$ There are two approaches for GBS screening and IAP: risk-based screening strategy and universal culture-based screening strategy. ${ }^{8}$ Universal culture-based screening strategy is defined as screening of all pregnant women for GBS infection by culture testing at 35-37 weeks' gestation, and decisions to administer IAP are based on a positive GBS culture. On the other hand, in risk-based screening strategy, IAP is administered based on risk factors that increase likelihood of early-onset GBS infection (ie, premature delivery at $<37$ weeks gestation, maternal fever at $>38^{\circ} \mathrm{C}$ and rupture of membranes for $>18$ hours). Under both strategies, IAP is recommended for women with GBS bacteriuria at any time during their current pregnancy or for women who had given birth previously to an infant with invasive EOGBS. ${ }^{8}$

The incidence of EOGBS in USA decreases from 1.7 cases per 1000 live births to 0.5 case per 1000 live births with the risk-based approach. ${ }^{8}$ However, the incidence decreased further to $0.3-0.4$ case per 1000 live births after the implementation of universal culture-based screening. ${ }^{8}$ For these reasons, the Centers for Disease Control and Prevention (CDC) and other professional bodies in the United States (American Congress of Obstetricians and Gynaecologists, and the American Academy of Paediatrics) recommend the universal culture-based screening approach since 2002. ${ }^{9-11}$ A recent study published in 2019, from US, showed further decrease in the incidence of EOGBS to 0.23 per 1000 live births in 2015. ${ }^{12}$

On the other hand, the Royal College of Obstetrician and Gynaecology in UK and other professional bodies outside North America recommends risk-based GBS screening strategy. ${ }^{13}$

In Saudi Arabia, there are no national guidelines on GBS screening strategy during pregnancy (ie, universal culture-based vs risk-based). So, the screening practices are variable among institutions. ${ }^{14}$ In Saudi Arabia, antenatal care takes place at different healthcare settings (ie, primary healthcare centres, private sectors or governmental hospitals). Pregnant women have the right to choose where to follow up during pregnancy. It is done by physicians, including general practitioners and specialised obstetric and gynaecologists.

There is little known about Saudi women's attitude, knowledge and acceptance of GBS screening. Therefore, the present study aimed to determine the current knowledge, practices and attitude of pregnant women towards GBS screening during pregnancy in Al-Madinah City, Saudi Arabia.

\section{METHODS}

\section{Study design}

This is a cross-sectional study conducted at Madinah Maternity and Children Hospital (MMCH), Al-Madinah, Saudi Arabia, from 1 May to 31 July 2018.

\section{Study participants}

A total of 450 pregnant women who were in the postnatal department at MMCH during the study period were invited to participate in the study. Women who did not speak Arabic or English were excluded. The agreement to complete the interview was considered as consent after verbal explanation of the nature and purpose of the study.

\section{Patient and public involvement statement}

No patients or public agency were involved in the research team. However, the study aimed to measure public awareness regarding GBS infections during pregnancy. Results of this study may influence public health stakeholders to take further actions to address issues related to GBS screening during pregnancy in Saudi Arabia.

\section{Study questionnaire}

Each participant was interviewed by one member of the research team using a validated survey from a previously published study. ${ }^{15}$ The questionnaire was divided into four sections: demographic data and obstetric history, knowledge assessment questions, awareness and previous experience with GBS screening, and attitude towards different GBS screening strategies (ie, universal culturebased strategy vs risk-based strategy).

Women's knowledge was assessed using six (true/ false) questions and two multiple choice questions that measure their knowledge on the risk, transmission mode, consequences and treatment of GBS infections. Participants' answers were rewarded with three points for each correctly answered question and zero for the incorrectly answered ones. The sum of these scores ranges from 0 to 24, which was converted into percentage. Knowledge score means, along with 25th, 50th and 75th percentiles, were used to describe knowledge outcome. As knowledge scores were normally distributed (online supplementary appendix table 1 and figure 1 ) and to analyse knowledge outcome using the binary logistic regression analysis, the $75 \%$ score was considered as the passing score based on the 75th percentile. Participants' awareness was assessed by a single (yes/no) question.

Participants' experience was assessed on the basis of following six items (yes/no questions):

- Previous or current GBS colonisation.

- Receiving information about GBS risk assessment during current pregnancy.

- Receiving information about GBS risk assessment during delivery.

- Request by caring healthcare worker to undergo GBS testing.

- Being tested for GBS after being requested.

- Receiving the test result after being done.

To simplify the multivariate binary logistic regression, the non-linear categorical principal components analysis was conducted on these six items using statistical data analysis software (SPSS V.21). Each item was considered as one exposure to GBS. Then, the exposure score was transformed into a scale range between 1 and 100 (structural 
equation modelling confirmatory factor analysis is shown in the online supplementary appendix, p2).

\section{Study procedure}

The questionnaire was filled during the interview by interviewers. The interviewers were three of the authors. After completing the first three sections of the questionnaire (ie, demographic data and obstetric history, knowledge assessment questions, awareness and previous experience with GBS screening), information about GBS and GBS screening strategies (ie, risk-based and universal culturebased GBS screening) were explained to the participants, including advantages and disadvantages of both strategies. Women's attitude towards different GBS screening strategies was assessed by direct question about which strategy they would prefer.

\section{Statistical analysis}

IBM statistical data analysis software, SPSS V.21, was used for analysis. Means and SDs were used to describe metric continuous variables (ie, knowledge scores) and percentages for categorical and binary variables (ie, demographic variables, obstetric variables, awareness and questions about previous experience with GBS screening).

Univariate chi-square test $\left(\chi^{2}\right)$ goodness-of-fit nonparametric test was employed to assess the distribution of the women's answers for statistically significant difference from equal (50\% correct answer: $50 \%$ incorrect answer chances). Pearson's products moments tests were used to assess the correlations between continuous variables.
The chi-square test $\left(\chi^{2}\right)$ test of independence was used to assess the association between categorical variables. One-way analysis of variance test was used to assess the multilevel categorical variables for mean differences on continuously measured variables.

Binary multivariate logistic regression analysis was employed to explore the individual and combined associations between the independent variables (women's demographics, obstetric history and past experiences score) and the dependent variables (participants' awareness of GBS and the odds of scoring more than the 75th percentile in GBS knowledge questions).

\section{RESULTS}

During the study period, a total of 377 out of 450 women (response rate $83.7 \%$ ) were enrolled and completed the interview. The demographic data and obstetric history of the women are shown in table 1.

The overall mean knowledge score for women was $59.8 \%(\mathrm{SD}=16.3)$. The $25 \mathrm{th}, 50$ th and 75 th percentiles for scores were $50 \%, 63 \%$ and $75 \%$, respectively (online supplementary appendix table 1). Thirty-five participants $(9.3 \%)$ scored more than the 75 th percentile. Table 2 shows univariate $\chi^{2}$ analysis of knowledge questions. Majority of women (62\%) had incorrect answers regarding meningitis as a complication of GBS infection in neonates $(\mathrm{p}<0.001)$. Of the women, $60.5 \%$ incorrectly believed that GBS is a sexually transmitted diseases

Table 1 Demographic and obstetric characteristics of the participants $(N=377)$

\begin{tabular}{|c|c|c|c|}
\hline Item & Measure & Frequency & Percentage \\
\hline \multirow{2}{*}{ Age (years) } & $25-34$ & 167 & 44.3 \\
\hline & 45 or older & 17 & 4.5 \\
\hline Employment & No & 325 & 86.2 \\
\hline \multirow[t]{4}{*}{ Educational level } & Less than high school & 48 & 12.7 \\
\hline & Completed high school & 101 & 26.8 \\
\hline & Postsecondary and still studying & 91 & 24.1 \\
\hline & Completed postsecondary & 125 & 33.2 \\
\hline \multirow{2}{*}{ Prenatal care provider } & Primary healthcare centres & 56 & 14.9 \\
\hline & Private hospitals & 193 & 51.2 \\
\hline \multirow[t]{3}{*}{ Specialty of the caring physicians } & None & 19 & 5 \\
\hline & General and family doctor & 36 & 9.5 \\
\hline & Gynaecologist & 322 & 85.4 \\
\hline \multirow[t]{2}{*}{ Reason for referral } & Not referred/visiting & 310 & 82.2 \\
\hline & Referred for further management from other centres & 66 & 17.6 \\
\hline
\end{tabular}


Table 2 Univariate analysis of the women's answers to the knowledge questions on GBS screening ( $N=377)$

\begin{tabular}{|c|c|c|c|}
\hline & Incorrectly answered & Correctly answered & $P$ value \\
\hline GBS is a leading cause of serious blood infections? & $151(40.1 \%)$ & $226(59.9 \%)$ & $<0.001$ \\
\hline GBS is a leading cause of meningitis? & $234(62.1 \%)$ & $143(37.9 \%)$ & $<0.001$ \\
\hline It is a sexually transmitted infection? & $228(60.5 \%)$ & $149(39.5 \%)$ & $<0.001$ \\
\hline It can be transmitted to a newborn during delivery? & $63(16.7 \%)$ & $314(83.3 \%)$ & $<0.001$ \\
\hline $\begin{array}{l}\text { GBS carrier mother cannot give birth through normal vaginal } \\
\text { delivery? }\end{array}$ & $133(35.3 \%)$ & $244(64.7 \%)$ & $<0.001$ \\
\hline GBS carrier mother cannot breast feed? & $58(15.4 \%)$ & $319(84.6 \%)$ & $<0.001$ \\
\hline $\begin{array}{l}\text { The risk of GBS being passed from mother to baby is highest } \\
\text { (during, before or after) delivery? }\end{array}$ & $112(29.7 \%)$ & $265(70.3 \%)$ & $<0.001$ \\
\hline $\begin{array}{l}\text { If a woman carries GBS during pregnancy, when she must get } \\
\text { antibiotics? }\end{array}$ & $232(61.5 \%)$ & $145(38.5 \%)$ & $<0.001$ \\
\hline
\end{tabular}

GBS, group B streptococcus.

$(\mathrm{p}<0.001)$. Also, about $60 \%$ of the participants did not know when antibiotic prophylaxis should be given for colonized or at risk pregnant mothers.

Multivariate binary logistic regression explaining the association between women's characteristics and their odds of passing a GBS knowledge score above the 75th percentile is shown in table 3 . Women with higher past exposure scores have significantly higher odds (OR 1.025, 95\% CI 1.009 to 1.039) of scoring above the 75th percentile in the knowledge score. For each additional point on the exposure to GBS screening score, the odds of women being informed on the GBS rises by 2.4\% ( $\mathrm{p}=0.001)$. Women's age converged significantly and negatively on scoring above the 75th percentile in the knowledge score (OR $0.28,95 \%$ CI 0.139 to 0.603 ) (online supplementary appendix figure B). Moreover, women's parity numbers correlate significantly with higher odds of scoring above the 75th percentile on knowledge score. The odds of scoring above the $75 \%$ score are predicted to rise by $13.71 \%$, on average, as the number of pregnancies increases by one $(\mathrm{p}=0.008)$. Pregnant women who were followed up by gynaecologists had higher odds of scoring more than the 75th percentile. However, it was not statistically significant $(\mathrm{p}=0.421)$.

The descriptive analysis of GBS awareness and exposure to GBS screening percentages is shown in table 4 .

Results of the $\chi^{2}$ test of association between the awareness of GBS screening and demographic and obstetric variables are shown in online supplementary appendix table 2. Multivariate logistic regression analysis of GBS awareness (Q8 in the questionnaire) and demographic and obstetric variables is shown table 5 . There was a statistically significant association between women's awareness of GBS screening and specialty of the healthcare professionals providing antenatal care. Women followed up by gynaecologists were significantly more aware of the GBS screening as compared with those cared for by other specialties $(\mathrm{p}=0.018)$.

Majority of the women $(61.8 \%)$ believed that the universal culture-based GBS screening strategy is better than risk-based strategy to prevent GBS infection in neonates. However, there was no statistically significant

Table 3 Multivariate binary logistic regression explaining the association between women's characteristics and their odds of passing the GBS knowledge score above (75th percentile)

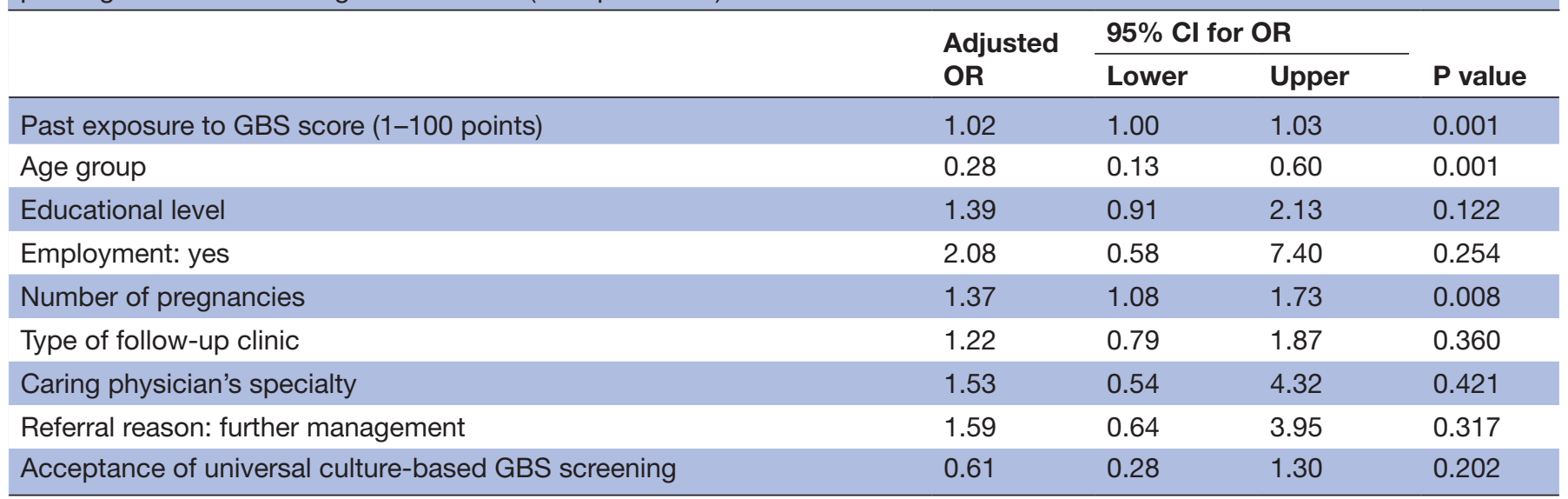

GBS, group B streptococcus. 
Table 4 Women's awareness and past experiences with GBS screening $(\mathrm{N}=377)$

\begin{tabular}{|c|c|c|c|}
\hline Item & Measure & Frequency & Percentage \\
\hline \multirow[t]{2}{*}{ Are you aware of GBS? } & No & 252 & 66.8 \\
\hline & Yes & 125 & 33.2 \\
\hline \multirow[t]{2}{*}{ Have you ever had GBS? } & No & 361 & 95.8 \\
\hline & Yes & 16 & 4.2 \\
\hline \multirow{2}{*}{$\begin{array}{l}\text { During current pregnancy, were you informed of GBS } \\
\text { risk assesment? }\end{array}$} & No & 329 & 87.3 \\
\hline & Yes & 48 & 12.7 \\
\hline \multirow[t]{4}{*}{ Who informed you of GBS risk for pregnant women? } & Never been informed & 329 & 87.3 \\
\hline & General practitioner/family doc & 6 & 1.6 \\
\hline & Gynaecologist & 37 & 9.8 \\
\hline & Nurses and other sources & 5 & 1.3 \\
\hline \multirow{2}{*}{$\begin{array}{l}\text { Are you aware of the available risk screening for GBS } \\
\text { during labour? }\end{array}$} & No & 326 & 86.5 \\
\hline & Yes & 51 & 13.5 \\
\hline \multirow{2}{*}{$\begin{array}{l}\text { Have you ever been requested to undergo GBS } \\
\text { testing? }\end{array}$} & No & 329 & 87.3 \\
\hline & Yes & 48 & 12.7 \\
\hline \multirow[t]{2}{*}{ Who requested you to undergo GBS testing? ( $n=48)$} & General practitioner/family doc & 5 & 10.4 \\
\hline & Gynaecologist & 43 & 89.6 \\
\hline \multirow[t]{2}{*}{ Did you undergo the requested GBS testing? ( $n=48)$} & No & 14 & 29.1 \\
\hline & Yes & 34 & 70.9 \\
\hline \multirow[t]{3}{*}{$\begin{array}{l}\text { When were you tested/requested to undergo GBS } \\
\text { testing during your pregnancy? }(n=48)\end{array}$} & $\begin{array}{l}\text { Below or during first } 30 \text { weeks of } \\
\text { pregnancy }\end{array}$ & 19 & 39.5 \\
\hline & After 30 weeks of pregnancy & 23 & 48 \\
\hline & Cannot remember & 6 & 12.5 \\
\hline \multirow[t]{2}{*}{ Were you informed of the GBS testing result? $(\mathrm{n}=34)$} & No & 6 & 17.6 \\
\hline & Yes & 28 & 82.4 \\
\hline
\end{tabular}

GBS, group B streptococcus.

Table 5 Multivariate logistic regression analysis of women's demographic and obstetric variables with their odds of being aware of GBS screening (Q8)

\begin{tabular}{|c|c|c|c|c|}
\hline & & $95 \% \mathrm{Cl}$ & & \\
\hline & Adjusted OR & Lower & Upper & $P$ value \\
\hline Age (years) & 0.89 & 0.58 & 1.35 & 0.599 \\
\hline Educational level & 1.28 & 0.99 & 1.65 & 0.057 \\
\hline Employment: yes & 1.76 & 0.80 & 3.87 & 0.156 \\
\hline Number of previous pregnancies & 0.50 & 0.29 & 0.87 & 0.014 \\
\hline Number of previous delivered babies & 2.05 & 1.14 & 3.67 & 0.016 \\
\hline Type of follow-up clinic & 0.94 & 0.71 & 1.23 & 0.670 \\
\hline Caring physician specialty: gynaecologist & 2.16 & 1.13 & 4.10 & 0.018 \\
\hline Past exposure to GBS score (1-100 points) & 2.12 & 1.50 & 3.00 & 0.000 \\
\hline GBS knowledge score (1-100 points) & 1.01 & 0.99 & 1.02 & 0.116 \\
\hline Sources of information & 1.33 & 0.96 & 1.84 & 0.082 \\
\hline Perceived best way of GBS prevention: universal screening, all women & 0.68 & 0.41 & 1.13 & 0.141 \\
\hline Constant & 0.15 & & & 0.129 \\
\hline
\end{tabular}

GBS, group B streptococcus. 
difference across demographic and obstetric variables regarding preference for the universal culture-based approach (online supplementary appendix table 3 ).

\section{DISCUSSION}

The present study has shown that majority $(66 \%)$ of the pregnant women were not aware of antenatal GBS screening strategies with vaginal and rectal swabs. Lower awareness regarding GBS compared with pertussis and influenza has been reported by McQuaid et al among women in a UK study. About $30 \%$ of women in that study never heard about GBS. ${ }^{16}$ However, higher awareness rate among women in the UK compared with women in the current study could be explained by the presence of national guidelines in the UK. Another study conducted in Hong Kong showed that only $36 \%$ of the participants had ever heard about GBS. ${ }^{17}$ In this study, only $12.7 \%$ of women were asked to do the GBS screening test, which was mainly requested by the gynaecologists.

Majority of pregnant women have poor knowledge about GBS infection and screening strategies, as $69.5 \%$ of them scored below $65 \%$ in the knowledge score. This result was consistent with previous studies conducted by Chow et al and Youden et al. ${ }^{15} 17$ The knowledge deficit about GBS infection and its outcomes may be due to lack of national guidelines on antenatal screening, which might result in variability in screening practices among physicians. ${ }^{14}$ Women with good knowledge about GBS were more likely to be cared for obstetric and gynaecologist physicians and were exposed to GBS. This might be explained by the lower rate of GBS testing requests among non-obstetric/gynaecologist healthcare professionals.

Knowledge deficit was more significant in terms of acknowledging the consequences of GBS infection among newborns, as most women did not know that GBS might cause meningitis. However, the Hong Kong study showed that the majority of the participants (89\%) overestimated the risk of EOGBS infection on newborns delivered by GBS colonised mothers. ${ }^{17}$ A previous qualitative study has assessed knowledge, perceptions and experiences of women regarding GBS and its screening. ${ }^{18}$ The results were in agreement with the present study as majority of the women had little knowledge about GBS screening strategies. It seemed that women were struggling to understand the meaning and implications of GBS infection for their babies as well as for themselves. ${ }^{18}$

Around $61.8 \%$ of the pregnant women in our study believed that the universal culture-based screening method would be the best way to prevent GBS infection in newborns, after they were informed about the different approaches to GBS screening. This finding was close to the Hong Kong study, where $81 \%$ of participants were willing to have universal culture-based GBS screening during pregnancy and $66 \%$ agreed that universal culture-based GBS screening should be implemented. ${ }^{17}$ Participants who had heard about GBS had higher scores on knowledge of GBS and better acceptance to its screening. ${ }^{17}$
A previous study conducted in Saudi Arabia found that the GBS colonisation rate in pregnant women in the third trimester ranges from $9.2 \%$ to $27.6 \% .{ }^{19}$ Moreover, a recently published prospective study from three hospitals in Arabian Gulf countries, including Saudi Arabia, showed that the overall incidence of EOGBS was 0.9 per 1000 live births, which is higher than current incidence in high-income countries. ${ }^{20}$ However, there are no nationwide surveillance data about rates of EOGBS, and these data are crucial to choose the GBS screening strategy (ie, risk-based vs universal culture-based).

This study has limitations. It is a cross-sectional survey study, which might have recall bias among participants regarding previous GBS screening strategies. Also, we did not interview the healthcare workers to explore the cause of low request rate for GBS screening. Another limitation is that the interviews were conducted in the postnatal department of only one hospital in Al-Madinah. As a consequence, this may not represent the whole Al-Madinah region, where about 200000 women are of childbearing age. However, MMCH is the largest women hospital in Al-Madinah region with 14000 deliveries per year. Future studies need to consider more hospitals and a larger sample size for obtaining generalisable results.

The present study is the first study conducted in Saudi Arabia to assess public awareness about GBS screening. It might influence public health stakeholders to conduct further studies about GBS screening and might help in establishing national guidelines regarding GBS screening.

\section{CONCLUSION}

The present study has assessed the knowledge, attitude and current practices of pregnant women towards GBS screening in Al-Madinah, Saudi Arabia. It was concluded that the level of awareness and knowledge about GBS among the pregnant women was relatively poor; however, majority of the pregnant women prefer universal culturebased screening. The results have depicted the need for nationwide research to ascertain the cost-effectiveness of either GBS screening strategies (ie, universal culturebased vs risk-based) based on the prevalence of maternal GBS colonisation, vertical transmission to neonates and rate of early-onset neonatal disease in different provinces of Saudi Arabia. The study results would be helpful in future development of a national guideline regarding GBS screening strategy and reinforcing women education about different GBS screening strategies during pregnancy.

Acknowledgements The authors thank the Canadian Centre for Vaccinology for its contribution in providing consent to reuse the survey.

Contributors The primary and corresponding author, AA, wrote the study protocol and the initial draft of the manuscript and prepared the survey. AA, SA and BA interviewed the patients for data collections and completed the data entry. $A A$ and BA completed the data analysis and reviewed the final version of the manuscript.

Funding The authors have not declared a specific grant for this research from any funding agency in the public, commercial or not-for-profit sectors.

Competing interests None declared. 
Patient consent for publication Not required.

Ethics approval The study approval was obtained from both the research ethics boards of the Ministry of Health in Al-Madinah and Taibah University, Al-Madinah, Saudi Arabia.

Provenance and peer review Not commissioned; externally peer reviewed.

Data availability statement № data are available.

Open access This is an open access article distributed in accordance with the Creative Commons Attribution Non Commercial (CC BY-NC 4.0) license, which permits others to distribute, remix, adapt, build upon this work non-commercially, and license their derivative works on different terms, provided the original work is properly cited, appropriate credit is given, any changes made indicated, and the use is non-commercial. See: http://creativecommons.org/licenses/by-nc/4.0/.

\section{ORCID iDs}

Amer Alshengeti http://orcid.org/0000-0001-6861-6934

Abdulsalam Alawfi http://orcid.org/0000-0002-9927-3898

\section{REFERENCES}

1 Borghesi A, Stronati M, Fellay J. Neonatal group B streptococcal disease in otherwise healthy infants: failure of specific neonatal immune responses. Front Immunol 2017;8:215.

2 Shane AL, Stoll BJ. Neonatal sepsis: progress towards improved outcomes. J Infect 2014;68 Suppl 1:S24-32.

3 Heath PT. Status of vaccine research and development of vaccines for GBS. Vaccine 2016;34:2876-9.

4 Creti R, Imperi M, Berardi A, et al. Neonatal group B Streptococcus infections. Pediatr Infect Dis J 2017;36:256-62.

5 Kowalska B, Niemiec KT, Drejewicz H, et al. [Prevalence of group B streptococcal colonization in pregnant women and their newborns based on the results of examination of patients in the Obstetric and Gynecology Department of the National Research Institute of Mother and Child--a pilot study]. Ginekol Pol 2003;74:1223-7.

6 Madrid L, Seale AC, Kohli-Lynch M, et al. Infant group B streptococcal disease incidence and serotypes worldwide: systematic review and meta-analyses. Clin Infect Dis 2017;65:S160-72.

7 Santhanam S, Arun S, Rebekah G, et al. Perinatal risk factors for neonatal early-onset group B streptococcal sepsis after initiation of Risk-based maternal intrapartum antibiotic Prophylaxis-A case control study. J Trop Pediatr 2018;64:312-6.
8 Verani JR, McGee L, Schrag SJ, et al. Prevention of perinatal group B streptococcal disease--revised guidelines from CDC, 2010. MMWR Recomm Rep 2010;59:1-36.

9 Centers for Disease Control and Prevention. Prevention of perinatal group B streptococcal disease revised guidelines from CDC, 2010 continuing education, 2010.

10 American College of Obstetricians and Gynecologists Committee on Obstetric Practice. ACOG Committee opinion no. 485: prevention of early-onset group B streptococcal disease in newborns. Obstet Gynecol 2011;117:1019-27.

11 Baker CJ, Byington CL, Polin RA, et al. Policy statementRecommendations for the prevention of perinatal group $B$ streptococcal (GBS) disease. Pediatrics 2011;128:611-6.

12 Nanduri SA, Petit S, Smelser C, et al. Epidemiology of invasive earlyonset and late-onset group B streptococcal disease in the United States, 2006 to 2015: multistate laboratory and population-based surveillance. JAMA Pediatr 2019;173:224-33.

13 Royal College of Obstetrician and Gynecology. Prevention of early onset neonatal group B streptococcal disease. London, UK: Royal College of Obstetricians and Gynaecologists (RCOG), Green-top guideline, 2012: 36. 1-13.

14 Musleh J, Al Qahtani N. Group B Streptococcus colonization among Saudi women during labor. Saudi J Med Med Sci 2018;6:18-22.

15 Youden L, Downing M, Halperin B, et al. Group B streptococcal testing during pregnancy: survey of postpartum women and audit of current prenatal screening practices. J Obstet Gynaecol Can 2005;27:1006-12.

16 McQuaid F, Jones C, Stevens Z, et al. Factors influencing women's attitudes towards antenatal vaccines, group B Streptococcus and clinical trial participation in pregnancy: an online survey. BMJ Open 2016;6:e010790.

17 Chow TY, William WK, Daniel TO. Knowledge and attitudes of Hong Kong pregnant women on group B Streptococcus screening. Department of Obstetrics and Gynaecology, United Christian Hospital, 2013.

18 Darbyshire P, Collins C, McDonald HM, et al. Taking antenatal group B Streptococcus seriously: women's experiences of screening and perceptions of risk. Birth 2003;30:116-23.

19 Al-Suleiman SA, Farrag I, Kingsley T-D, et al. Third trimester colonisation and treatment of group B $\beta$-haemolytic Streptococcus among obstetric patients in the eastern Province of Saudi Arabia. $J$ Obstet Gynaecol 1991;11:409-13.

20 Hammoud MS, Al-Taiar A, Al-Abdi SY, et al. Culture-Proven earlyonset neonatal sepsis in Arab states in the Gulf region: two-year prospective study. Int $J$ Infect Dis 2017;55:11-15. 